\title{
Population rates of cardiac catheterization and yield of high-risk coronary artery disease
}

\author{
Michelle M. Graham, William A. Ghali, Peter D. Faris, P. Diane Galbraith, Jack V. Tu, \\ Colleen M. Norris, Ali Zentner, Merril L. Knudtson; for the APPROACH Investigators
}

ß See related article page 49

Abstract

Background: The optimal population rate of cardiac catheterization is unknown. One potential way to determine it would be to examine whether there is a population rate beyond which the yield of high-risk coronary artery disease (CAD) does not rise.

Methods: Using a detailed clinical registry that captures all patients undergoing cardiac catheterization in Alberta, we determined annual population rates of cardiac catheterization and the corresponding yield of cases of high-risk CAD in each of Alberta's 17 health regions from 1995 to 2002. Least squares linear regression analysis and hierarchical modelling methods were then used to assess the linear relation between catheterization rates and rates of high-risk CAD.

Results: The age-adjusted average rate of cardiac catheterization among men ranged from 404.9 to 638.1 per 100000 population aged over 20 years. Among women, the average rate ranged from 171.8 to 314.0 per 100000 . For both sexes, increased regional rates of catheterization were associated with a linearly increasing yield of high-risk CAD, with no evidence of a plateau in yield when more procedures were performed. One additional case of high-risk CAD was identified for every 2.5 additional cardiac catheterization procedures performed among men, and for every 3.7 additional procedures performed among women.

Interpretation: The increasing yield of patients with high-risk CAD associated with increased regional population rates of cardiac catheterization, together with the absence of a plateau in yield, suggests that Alberta's population rates of cardiac catheterization are suboptimal to detect people with high-risk CAD.

CMAJ 2005;173(1):35-9

$\mathrm{O}$ ne of the primary purposes of cardiac catheterization is to identify patients with severe coronary artery disease (CAD), namely left main disease, 3vessel disease, or 2-vessel disease with involvement of the proximal left anterior descending artery. These important subgroups of patients are those in whom a survival advantage has been demonstrated with revascularization procedures..$^{1-3}$

Significant practice variations in the use of cardiac catheterization procedures have been demonstrated across national borders ${ }^{4,5}$ and across provincial borders in Cana- $\mathrm{da}^{6,7}$ and state borders in the United States. ${ }^{8}$ Variations across small regional areas have also been identified. ${ }^{9,10} \mathrm{Al}$ though there is some speculation that an ideal cardiac catheterization rate exists, we actually know little about what this ideal rate might be. One potential way to search for an optimal rate is to determine whether there is a population rate of cardiac catheterization beyond which the yield of high-risk CAD does not rise. Identifying such a plateau from cardiac registry data would be useful evidence toward the identification of an optimal catheterization rate.

The Alberta Provincial Project for Outcome Assessment in Coronary Heart Disease (APPROACH) is a large, population-based registry that captures detailed clinical information for all patients undergoing cardiac catheterization in Alberta. ${ }^{11}$ This database permits detailed study of the utilization of cardiac procedures at a population level. From the mid-1990s until Apr. 1, 2003, Alberta had 17 health regions, after which the province was reconfigured into 9 health regions. Given the existence of considerable variability in population rates of catheterization across these 17 regions, and across the years, we had a unique opportunity to study the relation between population rates of cardiac catheterization and the detection of high-risk CAD.

\section{Methods}

For the purpose of this study, APPROACH data from Jan. 1, 1995, to Mar. 31, 2002, were used. The database contains detailed clinical information, including demographic characteristics and comorbidities of patients, and tracks therapeutic interventions such as previous thrombolytic therapy and previous or subsequent revascularization procedures. Information on coronary anatomy is stored using a cardiac reconstruction software program (HeartView; Siemens Medical Systems). The extent of CAD is defined as the number of native coronary vessels with stenoses of more than $50 \%$; lesions in the proximal left anterior descending artery are considered separately. We defined high-risk CAD as left main disease, 3-vessel disease, or 2-vessel disease with involvement of the proximal left anterior descending artery.

In 2002 Alberta had a total population of 3.1 million and 17 
health regions (Fig. 1). Because of very small populations in some areas, we combined data for regions 14,15 and 17 for our analysis. The average number of people aged more than 20 years in the resulting 15 areas ranged from 26564 to 652536 (based on the average of populations from 1995 to 2002 [www.health.gov.ab.ca /regions/require/list.htm]). Only 2 of these areas (regions 4 and 10) have cardiac catheterization and revascularization facilities. These regions provide invasive investigation and revascularization for the entire province.

Separate statistical analyses were performed for men and women because the optimal population rate of cardiac catheterization may differ between sexes. We categorized patients by the health region in which they resided, according to the demographic information in the APPROACH database. Cardiac catheterization rates for each region were derived as the total number of catheterization procedures divided by the total population over age 20. Rates of high-risk CAD identified were calculated as the number of patients with high-risk CAD divided by the total population in the region over age 20. Cardiac catheterization rates for age categories over 20 were calculated for each region by dividing the total number of catheterization procedures within each age category by the population in that category. The age categories were $20-34,35-49,50-64,65-74$, and 75 and over. Rates of high-risk CAD for each region were calculated in the same manner. We then obtained indirectly standardized catheterization

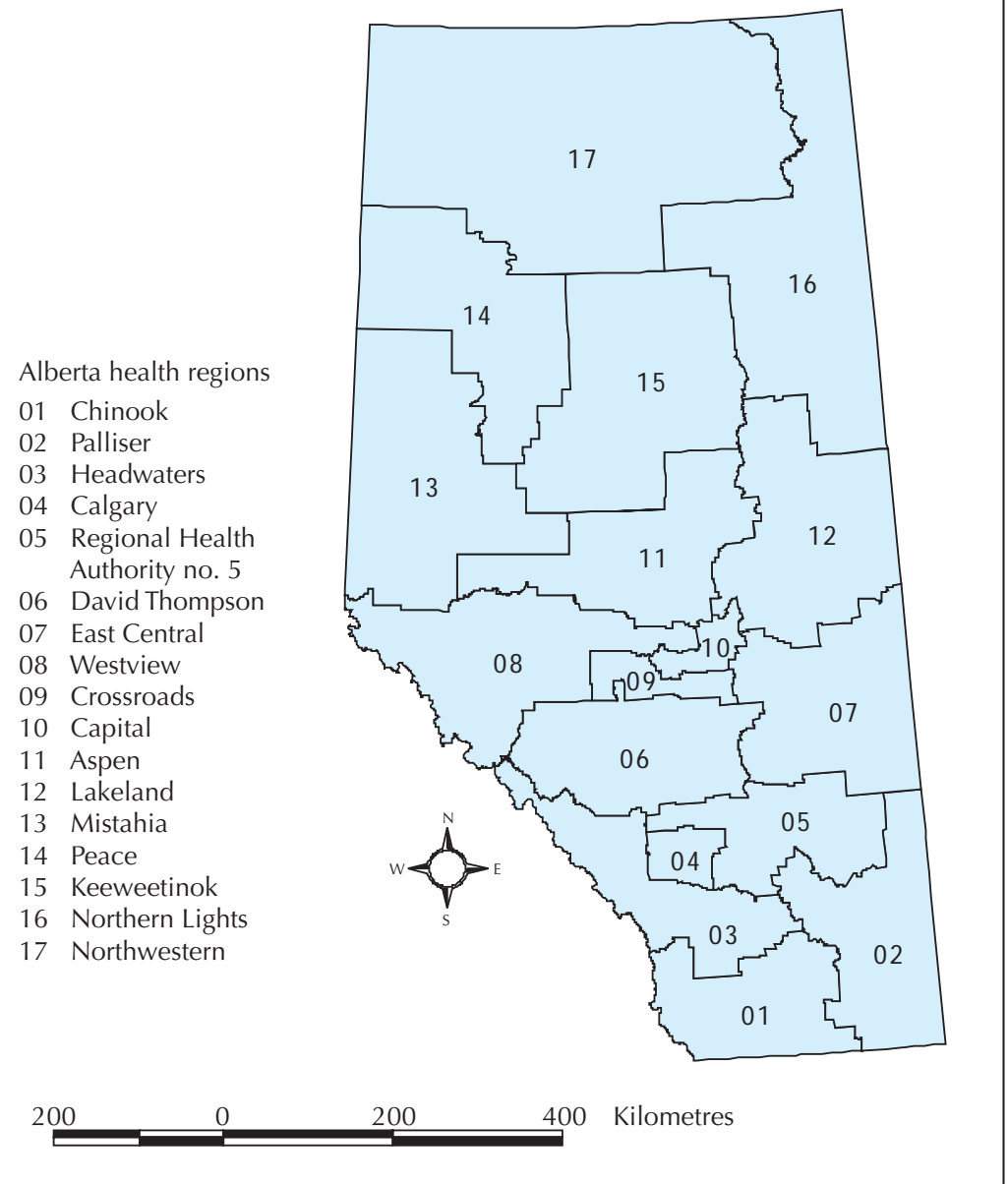

Fig. 1: Health regions in Alberta in 2002. rates and high-risk CAD rates per 100000 population aged over 20 by weighting the age-specific rates on the basis of the 1996 Canadian population. These adjusted rates were obtained separately for men and women.

A simple scatter plot was constructed of the annual catheterization rate versus the annual rate of high-risk CAD for each of the regions, with data points representing individual years. We then studied the relation between the catheterization rates and the high-risk $\mathrm{CAD}$ rates using least squares linear regression analysis, fitting straight lines between the 8 points for each health region. Because the observations from each region are not independent, and data from one region are more closely related to each other than to data from other regions, we also performed hierarchical modelling using a mixed-effects linear model, in which random effects were modelled for each region, with regional effects representing random variation around an average underlying relation. ${ }^{12}$ In this analysis, we first modelled a random slope and intercept for each region. We then compared this model with a more parsimonious model in which regions shared a common fixed intercept (fixed at zero) and random slopes. If there was no statistical evidence $(p<0.05)$ of the random intercepts model providing a better fit, the random slopes model (fixed intercept at zero) was accepted as the best representation of the data. The results of this accepted model were then used to plot a single weighted line that best reflected the linear relation between catheterization rate and high-risk CAD rate for all of the regions studied. This "best fit" line, with confidence intervals, was then presented on the original scatter plot.

To test whether we had evidence of a plateau in high-risk CAD rates at higher catheterization rates, we tested quadratic terms in each stage of the modelling. A statistically significant negative coefficient for the quadratic term in this latter analysis would indicate evidence of a plateau in the yield of high-risk CAD associated with increased rates of catheterization.

\section{Results}

Table 1 shows the average population of men and women over 20 years of age in each health region (based on the average of populations from 1995 to 2002) and the associated crude and adjusted average cardiac catheterization rates for each region. Among men, the adjusted catheterization rate ranged from 404.9 per 100000 adults over age 20 (region 7) to 638.1 per 100000 (region 4). The 2 regions with catheterization facilities (regions 4 and 10) had among the highest rates in Alberta. Most of the health regions had catheterization rates among men that were comparable, within the range of 475-575 per 100000 .

A different pattern emerged among women: their adjusted catheterization rate ranged from 171.8 per 100000 adults over age 20 (region 7) to 314.0 per 100000 (region 5). Most of the remaining health re- 
gions had rates in the range of $200-300$ per 100000 , significantly lower than the rates among men.

Fig. 2 demonstrates the relation between catheterization rate and yield of high-risk anatomy as a scatter plot containing data for each health region; individual points on the plot represent data for each year. For both men and women, higher cardiac catheterization rates were associated with higher yields of high-risk CAD.

The results of the least squares linear regression analysis are presented in Fig. 3. For men, the slope of the line was positive for all of the health regions (mean 0.35 , range $0.11-0.77$ ), which indicated a general tendency across regions for the yield of high-risk CAD to increase with increasing catheterization rates. For women, the slope was positive for all but 2 of the health regions (mean 0.197 , range -0.07 to 0.60 ).

The results of the hierarchical linear modelling are presented in Fig. 4. The final model was a mixed-effects model, in which the regions shared a common, fixed intercept at zero but had randomly varied slopes. This figure also includes an algebraic expression of the linear relation between catheterization rate and yield of high-risk CAD. For both men and women, the slopes are statistically different from zero $(p<0.001)$.

To statistically test for evidence of a plateau in the yield of high-risk CAD as catheterization rates increased, we formally tested for a quadratic term in our regression analysis. We found no significant coefficient, which implied no plateau effect. We therefore concluded that the yield of high-risk CAD in Alberta during the study period rose linearly with increasing cardiac catheterization rates.

On the basis of the slopes seen in the hierarchical modelling, for every 2.5 additional men and 3.7 additional women undergoing cardiac catheterization, about 1 additional patient with high-risk CAD is identified.

\section{Interpretation}

We found that, for each health region in Alberta, the yield of high-risk CAD increased linearly with an increase in cardiac catheterization rates and continued to do so without evidence of a plateau. This suggests that the optimal catheterization rate to detect high-risk CAD in Alberta is greater than 638.1 per 100000 adults over age 20

†Adjusted for age. among men and greater than 314.0 per 100000 among women. Given that rates of cardiac procedures in Alberta are among the highest in Canada ${ }^{6,7}$ our findings suggest that an optimal cardiac catheterization rate has not yet been reached in this country. These findings are of great international relevance because considerable rate differences exist across countries without evidence of optimal rates.

In comparison with other countries, Canada is considered to have a "medium" cardiac catheterization rate, simi-
Table 1: Average populations and average cardiac catheterization rates per 100000 population over 20 years of age in Alberta health regions

\begin{tabular}{|c|c|c|c|c|c|c|}
\hline \multirow{2}{*}{$\begin{array}{l}\text { Health } \\
\text { region }\end{array}$} & \multicolumn{2}{|c|}{ Population aged $>20 \mathrm{yr} *$} & \multicolumn{2}{|c|}{$\begin{array}{c}\text { Crude } \\
\text { catheterization rate }\end{array}$} & \multicolumn{2}{|c|}{$\begin{array}{c}\text { Adjusted } \\
\text { catheterization rate† }\end{array}$} \\
\hline & Male & Female & Men & Women & Men & Women \\
\hline 1 & 49274 & 51871 & 543.2 & 238.8 & 489.6 & 216.5 \\
\hline 2 & 31298 & 32008 & 515.5 & 238.4 & 487.8 & 223.7 \\
\hline 3 & 25593 & 25717 & 533.5 & 225.5 & 533.6 & 238.5 \\
\hline 4 & 321258 & 331278 & 580.8 & 269.4 & 638.1 & 305.5 \\
\hline 5 & 18195 & 18358 & 637.6 & 339.7 & 565.7 & 314.0 \\
\hline 6 & 62800 & 64382 & 608.2 & 278.4 & 589.2 & 280.8 \\
\hline 7 & 35245 & 36372 & 474.4 & 195.9 & 404.9 & 171.8 \\
\hline 8 & 30833 & 29954 & 511.9 & 199.8 & 524.5 & 234.3 \\
\hline 9 & 14161 & 14158 & 529.0 & 215.4 & 494.3 & 213.9 \\
\hline 10 & 287890 & 301414 & 544.2 & 221.9 & 542.0 & 228.6 \\
\hline 11 & 29502 & 28666 & 555.1 & 231.0 & 507.6 & 235.5 \\
\hline 12 & 33722 & 34895 & 656.2 & 264.3 & 577.8 & 256.7 \\
\hline 13 & 30083 & 28783 & 438.6 & 163.5 & 486.5 & 200.6 \\
\hline $14,15,17 \neq$ & 20763 & 19720 & 475.4 & 173.5 & 543.3 & 231.6 \\
\hline 16 & 13832 & 12732 & 415.3 & 154.4 & 631.5 & 287.0 \\
\hline
\end{tabular}

*Based on average of populations from 1995 to 2002.

$\ddagger$ These regions were combined because of their very small populations.

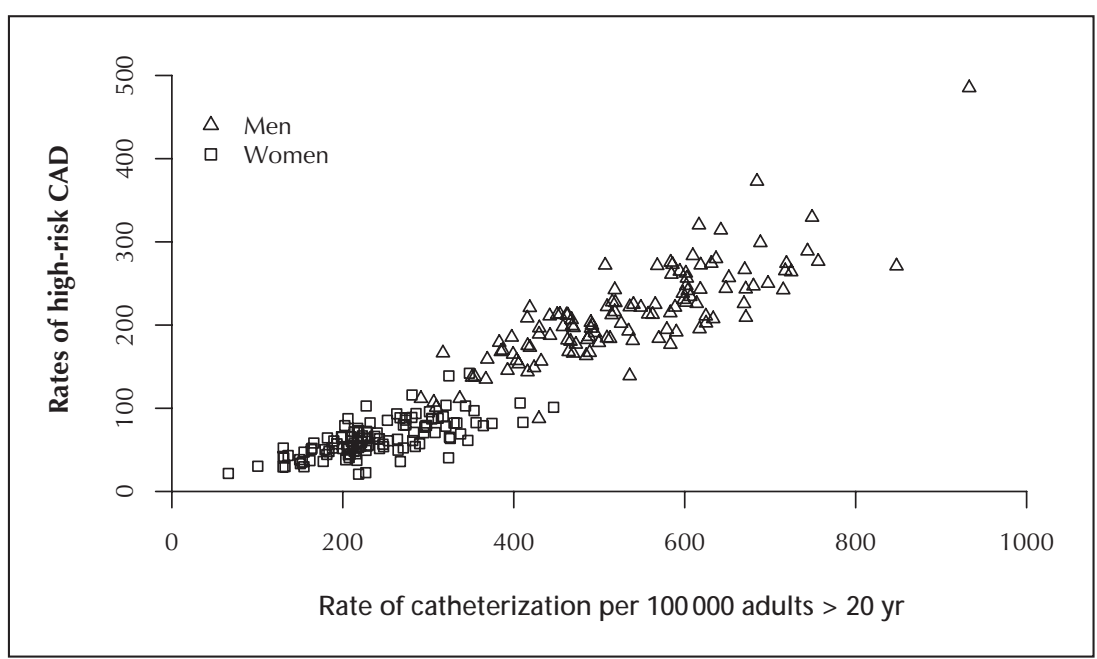

Fig. 2: Scatter plot of regional rates of high-risk coronary artery disease (CAD) versus rates of cardiac catheterization among men and women per 100000 population over 20 years of age. Each health region is represented by 8 data points (1995-2002). 
lar to that of Australia, Belgium and Germany, with lower rates reported in the Netherlands, Sweden, the United Kingdom, Hungary and Poland, and the highest rates in Brazil and the United States. ${ }^{413}$ Our results are consistent with those of a number of previous studies of cardiac procedure rates in Canada that indirectly suggested that a significant number of patients with high-risk CAD are not being identified. ${ }^{14,15}$ In a more direct comparison, Batchelor and colleagues ${ }^{16}$ determined that the rate of catheterization after myocardial infarction in the United States was more than 2.5 times that in Canada, with US physicians identifying on average 7.4 more cases of severe CAD per 100 postMI patients than Canadian physicians.

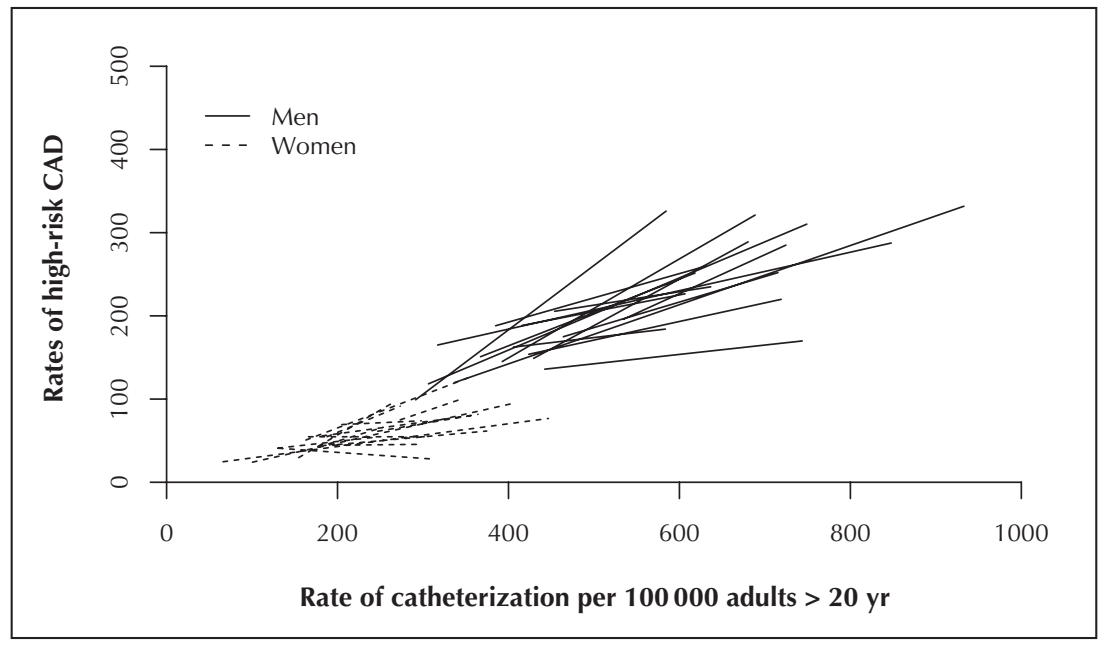

Fig. 3: Regression lines for each health region, derived from least squares linear regression analysis of rates of high-risk coronary artery disease (CAD) versus rates of cardiac catheterization per 100000 population over 20 years. For each region, the plotted line spans the range of cardiac catheterization rates.

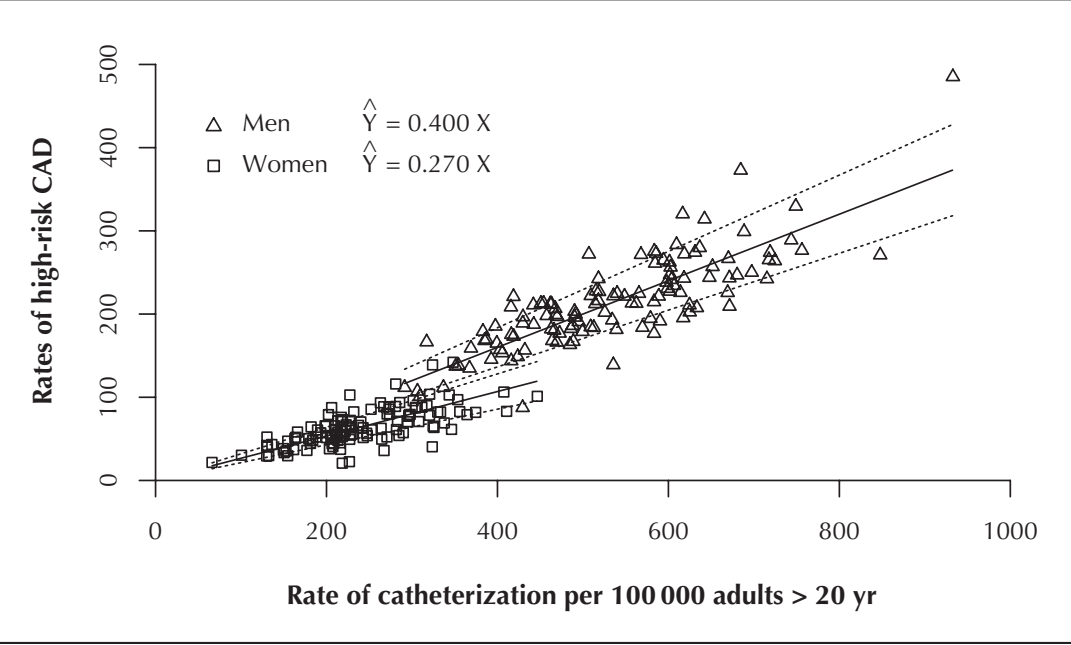

Fig. 4: Random slopes regression lines derived from hierarchical linear modelling using mixed-effects regression model. The 95\% confidence intervals (dotted lines) reflect variability in the mixed-effects slopes. The plotted lines span the range of cardiac catheterization rates among men and women.
In a previous study of APPROACH data, a large proportion $(74.2 \%)$ of the patients with high-risk CAD discovered at cardiac catheterization subsequently underwent revascularization procedures; in general, this tendency was uniform across regions. ${ }^{17}$ This finding indicates that the discovery of high-risk CAD in Alberta patients usually leads to therapeutic action (i.e., revascularization), which ay in turn improve long-term prognosis.

Implicit in some of the preceding discussion is that the Ise of catheterization is rational and truly linked to need. reality, this may not be the case. Decisions to perform catheterization procedures are typically made by individual physicians faced with individual patients. Such a one-onone perspective is often blind to the perspective of population-based needs that our study has attempted to address. Furthermore, this argument could be extended to the question of whether societal investment is best aimed at sick individuals (i.e., clinical interventions) as opposed to sick populations (i.e., population-based health promotion interventions). Rose ${ }^{18}$ acknowledged the role of intervention that targets both ends of this spectrum, although he pointed out that the returns may be greater for population-based interventions. In this regard, we acknowledge that our findings need to be complemented by future work assessing the economic implications of various catheterization utilization strategies.

Our study has limitations. Because the inception point of APPROACH is cardiac catheterization, and because no reliable data on regional prevalence of CAD are available, age adjustment of catheterization rates was the only method used to account for true regional differences in prevalance of severe CAD. Also, formal assessments of the appropriateness of the catheterization procedures were not conducted. However, it has been previously shown that inappropriate use of angiography does not explain geographic variations in procedure rates. ${ }^{19}$

In conclusion, we found a linear relation between cardiac catheterization rates and the yield of high-risk CAD in all of the 17 health regions in Alberta. Increasing the current rate of use of this procedure could lead to the detection of more patients with high-risk CAD who would then potentially benefit from revascularization. Because $\mathrm{Al}$ berta has one of the highest rates of cardiac catheterization in Canada, and because Canada is considered to have an intermedi- 
ate rate of cardiac catheterization compared with rates in other countries, these results suggest that a higher utilization rate may be required, not only in Canada but in many other countries with similar or lower catheterization rates. Whether an optimal rate of catheterization has been reached in countries with higher utilization rates (e.g., the United States) has yet to be determined, perhaps through the use of analyses similar to those presented here.

This article has been peer reviewed.

From the Departments of Medicine (Graham) and Faculty of Nursing (Norris), University of Alberta, Edmonton, Alta.; the Departments of Medicine (Ghali), Cardiovascular Sciences (Knudtson) and Community Health Sciences (Ghali, Faris, Galbraith), and the Centre for Health and Policy Studies (Ghali), University of Calgary, Calgary, Alta.; the Institute for Clinical Evaluative Sciences, and the Departments of Medicine and Public Health Sciences (Tu), University of Toronto, Toronto, Ont.; the Chinook Health Region (Zentner), Lethbridge, Alta.; and the Calgary office of the Alberta Provincial Project for Outcomes Assessment in Coronary Heart Disease (APPROACH) (Galbraith).

Merril Knudtson is the principal investigator of APPROACH.

\section{Competing interests: None declared.}

Contributors: Michelle Graham contributed to the analyses and to the writing of the manuscript. William Ghali, Jack Tu and Merril Knudtson were responsible for the study design and analyses. Peter Faris was responsible for statistical analysis. Colleen Norris, Diane Galbraith and Ali Zentner were responsible for data compilation and preliminary analysis. All of the authors participated in critically revising the manuscript and approved the final version.

Acknowledgements: Analyses for this paper were supported by the Canadian Cardiovascular Outcomes Research Team (CCORT), an interdisciplinary health research team funded by the Canadian Institutes of Health Research and the Heart and Stroke Foundation of Canada. William Ghali is supported by a Health Scholar Award from the Alberta Heritage Foundation for Medical Research, and he and Jack Tu are supported by Government of Canada Research Chairs in Health Services Research. Diane Galbraith is supported by a CCORT Student Fellowship and by TORCH (Tomorrow's Research Cardiovascular Health Professionals).

The Alberta Provincial Project for Outcome Assessment in Coronary Heart Disease (APPROACH) was funded in 1995 by a grant from the W. Garfield Weston Foundation, with ongoing support from Merck Frosst Canada Inc., Monsanto Canada Inc.-Searle, Eli Lilly Canada Inc., Guidant Corporation, Boston Scientific Ltd., Hoffmann-La Roche Ltd., Johnson \& Johnson Inc.-Cordis Corporation, the ProvinceWide Services Committee of Alberta Health and Wellness, the Libin Cardiovascular Institute of Alberta, and the Calgary Health Region and the Capital Health Authority.

\section{References}

1. Veterans Administration Coronary Artery Bypass Surgery Cooperative Study Group. Eleven-year survival in the Veterans Administration randomized trial of coronary bypass surgery for stable angina. N Engl 7 Med 1984;311:1333-9.

2. Varnuaskas E. Twelve-year follow-up of survival in the randomized European Coronary Surgery Study. N Engl 7 Med 1988;319:332-7.

3. Yusuf S, Zucker D, Peduzzi P, Fisher LD, Takaro T, Kennedy JW, et al. Effect of coronary artery bypass graft surgery on survival: overview of 10-year results from randomised trials by the Coronary Artery Bypass Graft Surgery Trialists Collaboration [published erratum in Lancet 1994;344:1446]. Lancet 1994;344:563-70.

4. Collins-Nakai RL, Huysmans HA, Scully HE. 23rd Bethesda conference: ac- cess to cardiovascular care. Task Force 5: Access to cardiovascular care: an international comparison. 7 Am Coll Cardiol 1992;19:1477-85.

5. Tu JV, Pashos CL, Naylor CD, Chen E, Normand SL, Newhouse JP, et al Use of cardiac procedures and outcomes in elderly patients with myocardial infarction in the United States and Canada. N Engl 7 Med 1997;336:1500-5.

6. Higginson LAJ, Naylor CD. Chapter 5: Rates of cardiac catheterization, coronary angioplasty and coronary artery bypass surgery in Canada. Can $\mathcal{F}$ Cardiol 1997;13(Suppl D):47-52.

7. Faris PD, Grant FC, Galbraith PD, Gong Y, Ghali WA; Canadian Cardiovascular Outcomes Research Team. Diagnostic cardiac catheterization and revascularization rates for coronary heart disease. Can 7 Cardiol 2004;20:391-7.

8. Center for the Evaluative Clinical Sciences, Dartmouth Medical School. The Dartmouth atlas of bealth care. Hanover (NH): The Center. Available: www .dartmouthatlas.org (accessed 2005 May 12).

9. Blustein J. High technology cardiac procedures: the impact of service availability on service use in New York State. FAMA 1993;270:344-9.

10. Wennberg DE, Kellett MA, Dickens JD, Malenka DJ, Keilson LM, Keller $\mathrm{RB}$. The association between local diagnostic testing intensity and invasive cardiac procedures. FAMA 1996;275:1161-4.

11. Ghali WA, Knudtson ML; APPROACH Investigators. Overview of APPROACH - the Alberta Provincial Project for Outcomes Assessment in Coronary Heart Disease. Can 7 Cardiol 2000;16:1225-30.

12. Pinheiro JC, Bates DM. Mixed-effects models in S and S-PLUS. New York: Springer-Verlag; 2000.

13. Yusuf S, Flather M, Pogue J, Hunt D, Varigos J, Piegas L, et al. Variations between countries in invasive cardiac procedures and outcomes in patients with suspected unstable angina or myocardial infarction without initial ST elevation. Lancet 1998;352:507-14.

14. Alter DA, Austin P, Tu JV. Use of coronary angiography, angioplasty and bypass surgery after acute myocardial infarction in Ontario. In: Naylor CD, Slaughter PM, editors. Cardiovascular bealth and services in Ontario: an ICES atlas. Toronto: Institute for Clinical Evaluative Sciences; 1999. p. 141-64.

15. Tu JV, Naylor CD, Kumar D, DeBuono BA, McNeil BJ, Hannan EL. Coronary artery bypass graft surgery in Ontario and New York State: Which rate is right? Ann Intern Med 1997;126:13-9.

16. Batchelor WB, Peterson ED, Mark DB, Knight JD, Granger CB, Armstrong $\mathrm{PW}$, et al. A comparison of US and Canadian cardiac catheterization practices in detecting severe coronary artery disease after myocardial infarction: efficiency, yield and long-term implications. 7 Am Coll Cardiol 1999;34:12-9.

17. Seidel JE, Ghali WA, Faris PD, Bow CJ, Waters NM, Graham MM, et al; APPROACH Investigators. Geographical location of residence and uniformity of access to cardiac revascularization services after catheterization. Can $\mathcal{F}$ Cardiol 2004;20:517-23.

18. Rose G. Sick individuals and sick populations. Int 7 Epidemiol 1985;14:32-8.

19. Chassin MR, Kosecoff J, Park RE, Winslow CM, Kahn KL, Merrick NJ, et al. Does inappropriate use explain geographic variations in the use of health care services? FAMA 1987;258:2533-7.

Correspondence to: Dr. Michelle M. Graham, Division of Cardiology, University of Alberta Hospital, 8440-112 St., Edmonton AB T6G 2R7; fax 780 407-1496;

mmgraham@cha.ab.ca

\section{APPROACH Clinical Steering Committee}

Edmonton: Stephen Archer, Michelle M. Graham, William Hui,

Arvind Koshal, Colleen M. Norris and Ross T. Tsuyuki (chair);

Calgary: Michael J. Curtis, P. Diane Galbraith, William A.

Ghali, Merril L. Knudtson, Andrew Maitland, L. Brent Mitchell

and Dean Traboulsi 